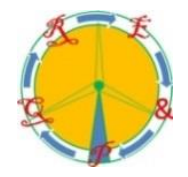

\title{
A Power Take-Off and Control Strategy in a Test Wave Energy Converter for a Moderate Wave Climate
}

\author{
K. L. De Koker ${ }^{1}$, G. Crevecoeur ${ }^{1}$, B. Meersman ${ }^{1}$, M. Vantorre $^{2}$ and L. Vandevelde ${ }^{1}$ \\ ${ }^{1}$ Department of Electrical Energy, Systems and Automation \\ Ghent University \\ Technologiepark-Zwijnaarde 913, 9052 Ghent, Belgium \\ Phone number:+32 9264 5694, e-mail: Kristof.DeKoker@UGent.be \\ ${ }^{2}$ Maritime Technology Division \\ Ghent University \\ Technologiepark-Zwijnaarde 904, 9052 Ghent, Belgium
}

\begin{abstract}
The energy in the waves of oceans and seas can be converted to electricity by different types of Wave Energy Converters (WECs). Wave energy conversion is currently widely studied to contribute to the world's rising energy needs. This paper describes a point absorber test WEC that was built for electrical energy production in moderate wave climates as can be found in the Belgian part of the North Sea. A robust design was put forward to assess the feasibility of a full electric rotational Power Take-Off (PTO) system. A stable reactive control algorithm was implemented to optimise the absorbed energy from the waves by tuning the natural frequency of the WEC towards the frequency of the waves. From simulations it is shown that also for real irregular waves, this tuning shows a significant beneficial effect on the absorbed energy. The control parameters for different wave conditions are discussed as well as the effect of the chosen PTO system and its constraints on the absorbed power and optimum control parameters.
\end{abstract}

\section{Key words}

Wave Energy Converter, Point Absorber, Supplementary mass, Electric PTO

\section{Introduction}

The absorption and conversion of ocean wave energy has a large potential to contribute to the world's electrical energy needs once the technology has reached maturity to technically and economically compete on the market. In the meantime, several concepts of Wave Energy Converters (WECs) are being researched and tested around the world. Three main classes of WECs can be distinguished: oscillating water column, oscillating bodies, and overtopping devices [1]. The test buoy described here is an oscillating body and more specifically a point absorber, characterised by a diameter that is relatively smallcompared to the incident wavelength.

The FlanSea consortium of university research groups and six companies designed and built a test WEC of $4.4 \mathrm{~m}$ diameter, baptised "Wave Pioneer", and laid out at sea for several months of testing. The project focussed on wave energy conversion in a moderate wave climate, as can be found in the Belgian part of the North Sea where an average wave power of $4.6 \mathrm{~kW} / \mathrm{m}$ wave crest is available [2].

The resulting Wave Pioneer is a point absorber type consisting of a buoy connected to the seabed by a cable. In the buoy, the cable is wound onto a drum and connected with an electrical Power Take-Off (PTO) system as depicted in Fig. 1. Two electrical machines are connected to the drum by means of a gearbox to increase the working torque and decrease the speed towards the drum. When the buoy is pushed upwards by the wave motion, the cable is wound off and electricity is generated by applying a torque in opposite direction with the electrical machines. During the downward movement, the electrical machines act as motor to wind up the cable and keep it under tension.

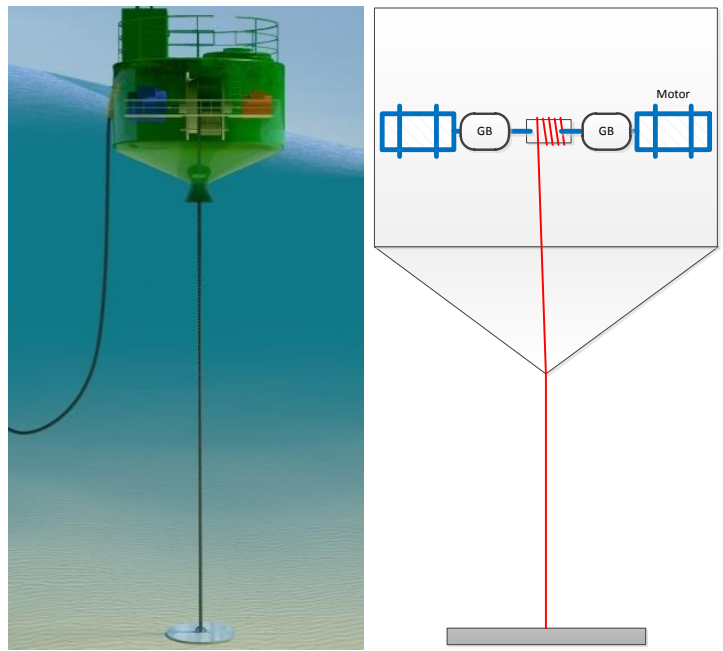

Fig. 1 Artist impression of the Wave Pioneer and schematic diagram of its PTO system with gearbox (GB) and motor - CFlanSea 
Point absorbers moving relative to the seabed can also consist of a hydraulic PTO such as in [3], or a linear generator such as in [4], with the additional difference that the generator unit is located on the seabed. The relative movement between two floating bodies can also act as energy source: a linear generator between a deep-draught spar and buoy is for instance used in [5]. Other point absorbers with a rotary generator can be found in [6], with the difference that a rack and pinion transmission is used instead of a cable. The control strategy of [6] is comparable to the one used in this paper as it also makes use of a damping coefficient and a mass coefficient, however simulations in regular wave are used to define optimal control parameters for irregular waves in contrast to the method described here.

To develop the Wave Pioneer, the project team studied and designed all necessary features to deploy the test buoy at sea, taking into account the conditions of the location: the buoy shape and structure, the cable, a survival system for storm conditions, and the PTO design. This paper focusses mainly on the implementation of a PTO and control strategy to optimise the absorbed power.

\section{Buoy shape and anchor}

As depicted in Fig. 1 the buoy has a conical shape with a cylindrical extension around the still water line. A floating buoy on the water acts as a spring-mass system with the hydrostatic force acting as the spring force. The cylindrical shape of the buoy around the water line creates a linear relationship between the vertical buoy position in relation to the still water line and the hydrostatic force.

Simulations have shown that buoy diameters of 8 to 10 meter could increase the economic viability. However, at this stage of research, a diameter of $4.4 \mathrm{~m}$ was chosen to create a real-life lab test buoy at reasonable scale to gain experience with the technology. The buoy weighs around 25 tons and the cable is attached to a 36 ton gravity based anchor.

\section{The PTO drive train}

The complete PTO drive train is schematically presented in Fig. 2 comprising a drum on which the cable is wound, two gearboxes and two electrical machines connected with two variable speed drives. The installed PTO power was split up in two machines to permit a high installed power to maximise research possibilities during the tests at sea while maintaining symmetry in the weight load in the WEC at the same time.

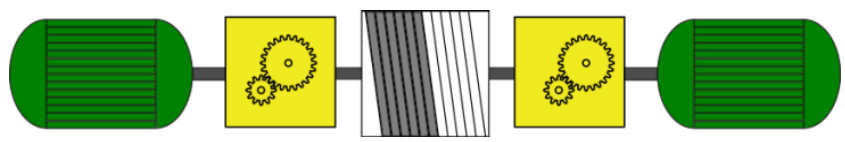

Fig. 2 Schematic presentation of the PTO drive train consisting of two electrical machines connected with a gearbox to the drum

The diameter of the drum should not be smaller than the minimum allowed bending radius of the cable. The gearboxes increase the torque applied by the electrical machines towards the drum. In addition, the relatively slow rotational speed of the drum is increased to enable the use of 1500rpm rated machines.
As the required PTO action is intermittent due to the motion of the waves, good engineering practice allows the usage of the machine in over-rated torques. Hence, the machine is used up to $200 \%$ of its rated torque and its RMS torque is monitored such that it stays below the rated value. This allows a reduction of the installed power of $50 \%$ in relation to usage up to the rated torque.

\section{The PTO control}

\section{A. Spring-mass principle}

As earlier introduced, a heaving buoy on the water acts as a spring-mass system. Moreover, due to the incident wave forces, it can be seen as an excited spring-mass system with the PTO as external damper. Indeed, by damping the buoy's motion, the PTO can extract energy from the waves. From such a system it is known that the maximum of energy can be extracted from the exciting force when the system is in resonance and by applying external (PTO) damping equal to the internal hydrodynamic damping. To reach resonance, the natural frequency $\omega_{\mathrm{n}}$ of the springmass system has to be equal to the frequency $\omega$ of the wave, assuming a regular wave. The natural frequency is expressed by:

$$
\omega_{\mathrm{n}}=\sqrt{\frac{k}{\left(m_{\text {buoy }}+m_{\mathrm{a}}\left(\omega_{\mathrm{n}}\right)\right)}}
$$

with $m_{\text {buoy }}$ the mass of the buoy, $m_{\mathrm{a}}\left(\omega_{\mathrm{n}}\right)$ the added mass for the natural frequency, $k$ the spring constant or hydrostatic restoring coefficient. The latter is expressed as $k=\rho g A_{\mathrm{w}}$, where $A_{\mathrm{w}}$ is the waterline area, $g$ the gravity constant, $\rho$ the density of the water. The added mass is the defined as the coefficient with which the buoy's vertical acceleration has to be multiplied to obtain the acceleration dependent component of the hydrodynamic reaction force, and can therefore be interpreted as the mass of the water surrounding the buoy and moving along with it.

As the incoming waves on a WEC cannot be controlled, two possible interventions to tune the natural frequency would be to change either the mass of the system, or the diameter of the buoy to influence the spring constant. However, these two interventions are physically difficult to accomplish, certainly when aiming at controlling the natural frequency to react on variable incoming waves. Nonetheless, a control strategy could consist of adding a supplementary mass $M_{\text {sup }}$ [7]. To overcome the difficulty of adding a physical mass, the supplementary mass is virtualised by applying a PTO force which is proportional with the buoy's vertical acceleration (here along the $z$ axis). As this force tunes the natural frequency of the system towards the frequency of the waves, it is called the tuning force $F_{\text {tun }}$ :

$$
F_{\text {tun }}=-M_{\text {sup }} \frac{d^{2} z}{d t^{2}}
$$

This results in an adaption of the natural frequency of (1) to 


$$
\omega_{\mathrm{n}}=\sqrt{\frac{k}{\left(m_{\text {buoy }}+m_{\mathrm{a}}\left(\omega_{\mathrm{n}}\right)+M_{\text {sup }}\right)}} .
$$

When neglecting all other modes of motion except the vertical translation, a damping force $F_{\text {damp }}$ proportional with the vertical velocity of the buoy can be applied by the PTO with external damping coefficient $B_{\text {ext }}$ :

$$
F_{\text {damp }}=-B_{\text {ext }} \frac{d z}{d t}
$$

\section{B. Control of a buoy with cable}

In the Wave Pioneer's case, the only way to exert a PTO force on the WEC is through the cable providing only downward forces on the buoy. Consequently the tuning force can only be applied when the acceleration is positive (i.e. directed upwards) and the damping force when the velocity is positive. Additionally, an extra force $F_{\text {tense }}$ should ensure that the cable remains under tension at all times. The total resulting PTO force can thus be expressed as:

$$
F_{\text {PTO }}=\left.F_{\text {damp }}\right|_{\frac{d z}{d t}>0}+\left.F_{\text {tun }}\right|_{\frac{d^{2} z}{d t^{2}}>0}+F_{\text {tense }}
$$

\section{Effect of control parameters on absorbed power}

Even though the philosophy of the described control strategy is based on the response of the system excited by regular waves, and irregular waves occurring in real seas cannot bring the WEC in resonance, an optimum combination of the parameters $B_{\text {ext }}$ and $M_{\text {sup }}$ can be found for irregular waves by running iterative simulations.

In real seas, the waveform can be considered as the sum of a large number of regular components of different amplitudes and periods. Various wave spectra can be measured at different times and can be identified by their significant wave height $H_{\mathrm{s}}$ and mean period $T_{\mathrm{m}}$. They can be divided in ranges of significant wave height called sea states (SS).

A time domain simulation model was built to estimate the absorbed power per sea state by calculating the hydrodynamic response of the WEC. Iterations of the simulation are run to make power plots as a function of the control parameters $B_{\text {ext }}$ and $M_{\text {sup }}$ [8].

A first simulation has been performed under the assumptions that an unlimited PTO force is available, the desired force (5) can always be applied and the PTO inertia is set to zero. For each sea state a multitude of combinations of values for $M_{\text {sup }}$ and $B_{\text {ext }}$ are simulated by iteratively changing them within a predefined range. The results are visualised in the colour plots of Fig. 3 where the absorbed power versus the control parameters $M_{\text {sup }}$ and $B_{\text {ext }}$ is plotted per sea state.

The Wave Pioneer buoy has a freeboard of two meter above the still water line. The distance between the still water line on the buoy and the actual water level is defined as sinkage. A positive sinkage is achieved when the buoy is pulled below the still water line. During operation, the buoy may never be overtopped by the water, i.e. a sinkage larger than $2 \mathrm{~m}$, as this will heavily affect the upwards movement of the buoy and is not desirable from operational point of view. Therefore a black contour plot indicates when the sinkage exceeds $2 \mathrm{~m}$ during the simulation. Results in Fig. 3 with a sinkage higher than $2 \mathrm{~m}$ are of no physical significance as the simulation model does not account for limitations of the height of the freeboard, and are therefore omitted.

The maximum absorbed power that can be reached is marked with a blue circle in each of the plots. The coordinates of the blue donut correspond with the optimal values for $M_{\text {sup }}$ and $B_{\text {ext }}$. As the sea state changes, these values differ, as plotted in Fig. 4. It shows the importance of using the appropriate parameters to prevent exceeding the maximum sinkage, and not at least to optimise the energy yield

The effect of the tuning in contrast with pure damping, can be appraised by comparing the maximum reachable power corresponding with $M_{\text {sup }}$ equal to zero in the plots. It is clear from these results, plotted in Fig. 5, that a substantial increase in absorbed power is reached by this means of reactive control.
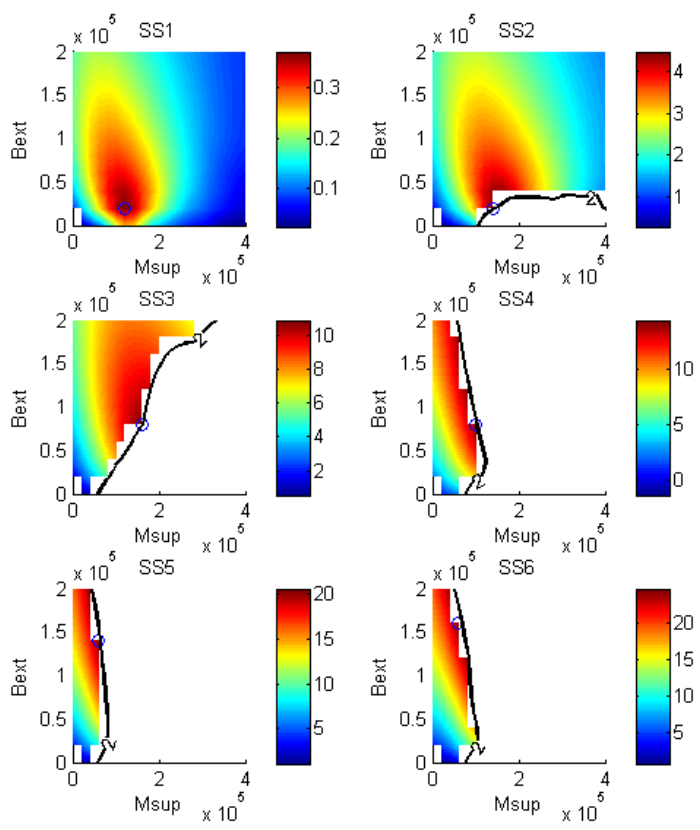

Fig. 3 Absorbed power [kW] vs. control parameters $M_{\text {sup }}$ and $B_{\text {ext }}$ for an unlimited available PTO force, contours for maximum sinkage (black)

It can be observed from the location of the blue donuts in the colour plots that the optimal control parameters differ significantly per sea state. An overview is given in Fig. 4.

The importance of applying the correct control parameters when a certain sea state occurs is clear in the plots of Fig. 3. Wrong parameters can result in exceeding the maximum sinkage, but also in a drastic drop in absorbed power.

Fig. 5 plots the maximum values of the absorbed power per sea state with the optimal control parameters that can be distilled from Fig. 3 for tuning and pure damping. 


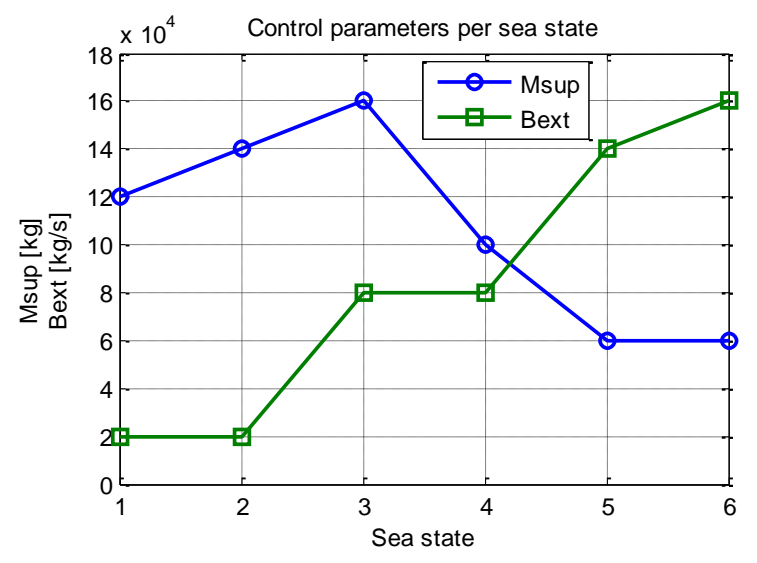

Fig. 4 Optimal control parameters $M_{\text {sup }}$ and $B_{\text {ext }}$ for an unlimited available PTO force

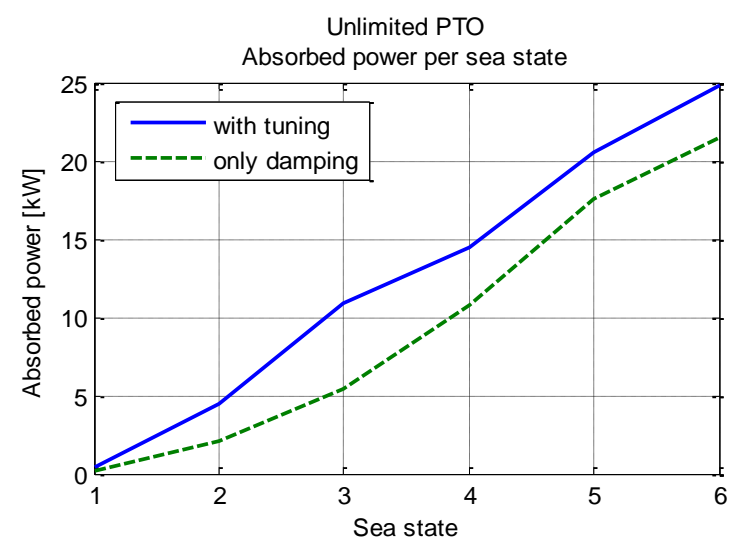

Fig. 5 Absorbed power per sea state for an unlimited PTO force with tuning (blue solid line) and only damping (green dashed line)

\section{Effect of the PTO constraints on the control parameters and absorbed power}

When a PTO topology and installed power is chosen, the corresponding constraints such as maximum allowed torque are included in the simulation model. Another constraint is the protection against overload, because the machine is used up to $200 \%$ of its rated torque, the RMS torque should remain below the nominal torque value. This monitoring is not done in the simulation itself, but in post-processing in the colour plots by means of a contour line.

The PTO constraints influence the maximum mean power that can be absorbed, and a shift in optimum parameters can be observed. A sensitivity analysis for the installed power has been executed with the hydrodynamic simulation model for the four most energetic sea states under study. Simulations were run with specifications of off-the-shelf available induction machines for six different installed powers P1 to P6 where the installed power of P6 is six times larger than P1, as shown in Table I.

Table I. - Installed powers of sensitivity analysis

\begin{tabular}{cc}
\hline Installed power & Approximate ratio to P1 \\
\hline P1 & 1 \\
P2 & 2 \\
P3 & 3 \\
P4 & 4 \\
P5 & 5 \\
P6 & 6 \\
\hline
\end{tabular}

The higher the installed power is, the higher the maximum applicable torque and thus the applicable PTO force on the cable. The desired value of the PTO force (5) at every moment will be limited if it would exceed the maximum torque limit of the machine. The results collected in Fig. 6 illustrate the sensitivity of the available PTO force, i.e. installed power, on the absorbed power from the waves. It is clear that cases P1 and P2 miss a large share of the wave power. Case P3 matches approximately with the absorbed power of an unlimited PTO in sea state 3 and 4 . Case P4 and above match in sea state 3 and even exceed the unlimited PTO at sea states 4 and higher. This is likely due to the realistic PTO inertia in the simulation for P1 to P6 in contrast to no inertia in the unlimited case, or due to the parameter step size in the iterations.

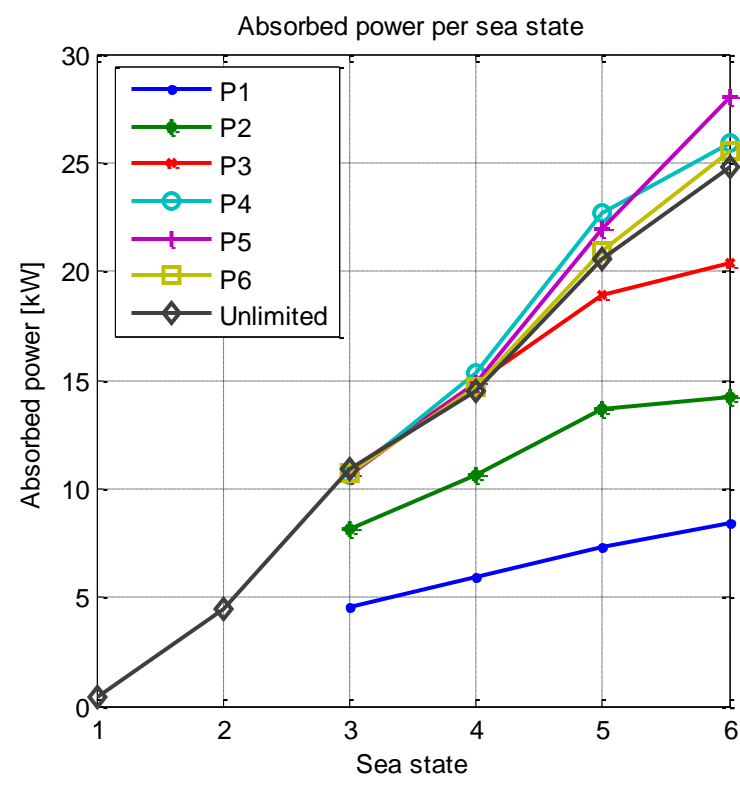

Fig. 6 Absorbed power per sea state for different installed powers

When the only limit in the colour plots of Fig. 3 was the sinkage (black contour), in Fig. 7 the RMS torque of the machine is also observed. The magenta contour line in the plots defines the limit of the used machine to prevent overload.

Analysis of Fig. 7 shows that the lower applicable PTO force with the low installed power of P2 limits the maximum mean power that can be absorbed in comparison with the larger machines P3 and P5. Even without the RMS torque limit, the maximum mean power with a P2 machine remains far below the maxima of higher installed powers, as can be read from the maxima of the coloured legend bars. When the installed power rises further, the control parameters are no longer determined by the RMS torque limit, but again by the maximum allowed sinkage as was the case with the unlimited PTO.

For the Wave Pioneer, the installed power of P3 was chosen. This PTO enables ample research possibilities as the simulations illustrate its ability to absorb the maximum achievable power up to sea state 4 . 


\section{Conclusion}

The presented WEC resulted in a test device enhancing research opportunities for tests at sea. The PTO and its control are conceived to enable high flexibility during the tests. The reactive control to tune the natural frequency of the WEC towards the frequency of the waves is intelligible providing a test device to gain maximum experience in the field and expose the sensitivities of the technology for further research and development.

Appropriate choice of the values for the control parameters is key to optimise the energy yield. The optimal values are dependent on the wave conditions.

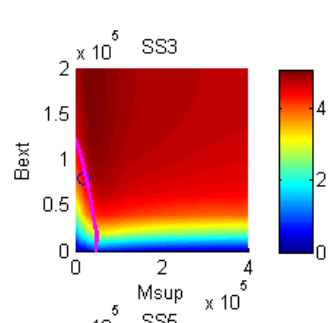

P1
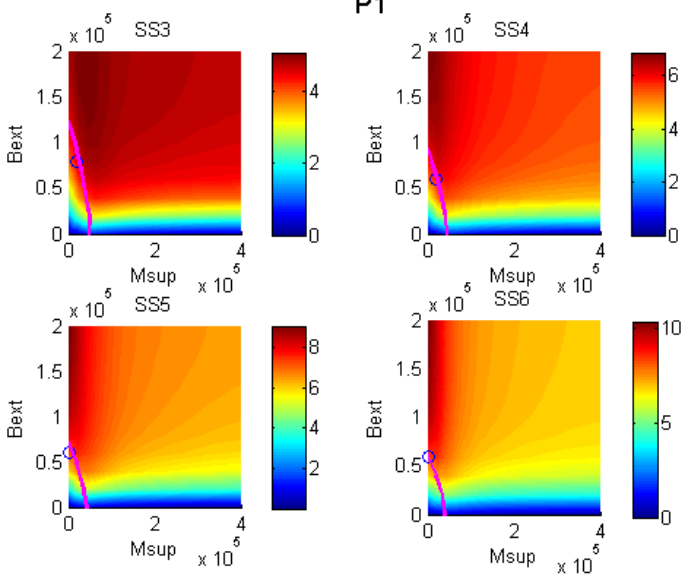

P3
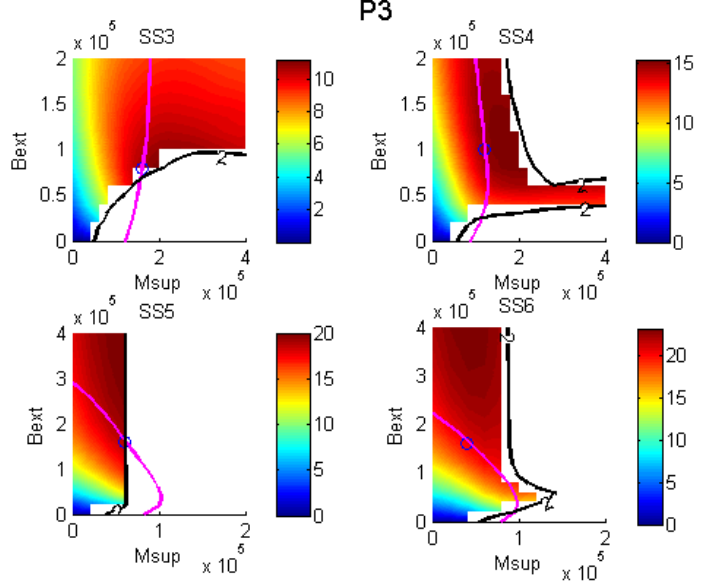

P5
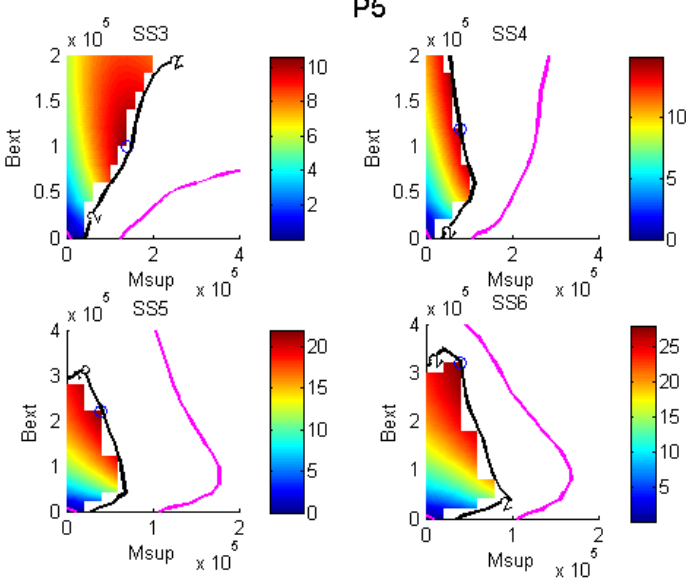

The installed power and the corresponding PTO constraints of maximum and RMS torque have a substantial influence on the achievable mean absorbed power. An extensive techno-economical study taking account for the location, and thus the occurrence frequencies of each sea state, is necessary to decide on the ideal installed power of a WEC for a certain location. For the Wave Pioneer, the installed power was chosen based on research criteria rather than economic optimisation.

\section{Acknowledgement}

A part of the work presented in this paper was carried out during the FlanSea project, financed by the Agency for
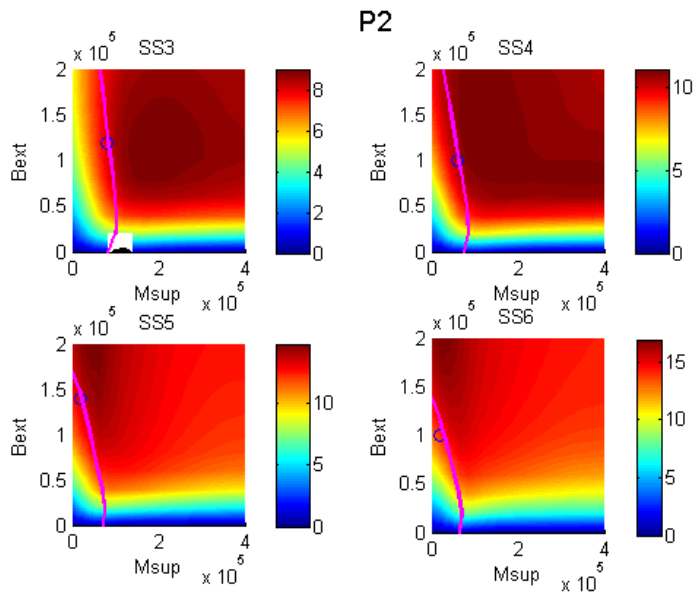

P4
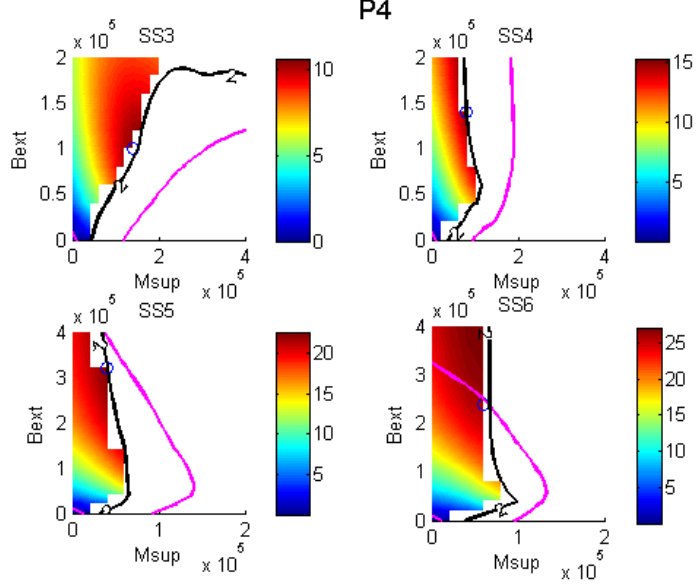

P6
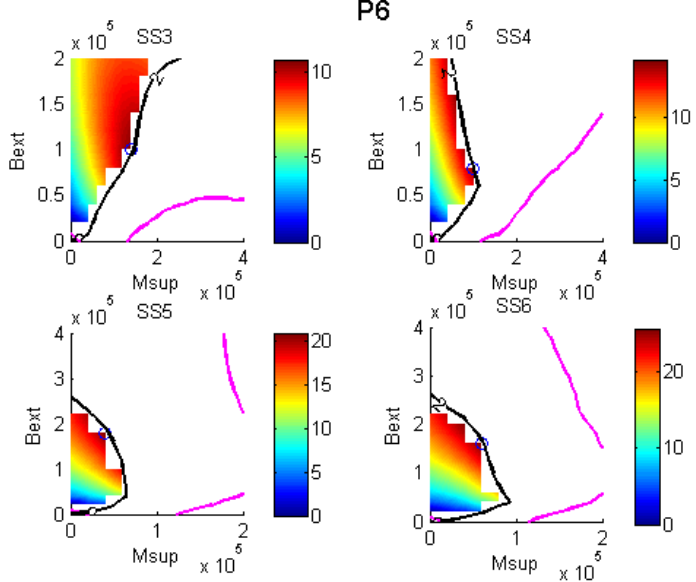

Fig. 7 Absorbed power [kW] vs. control parameters $M_{\text {sup }}$ and $B_{\text {ext }}$ for three different installed powers for SS3-6, contours for maximum sinkage (black) and RMS torque (magenta) 
Innovation by Science and Technology in Flanders (IWT) and the industrial partners DEME Blue Energy, Cloostermans, Spiromatic, Port of Oostende, Electrawinds and Contec.

\section{References}

[1] A. F. D. Falcao, "Wave energy utilization: A review of the technologies," Renewable \& Sustainable Energy Reviews, vol. 14, no. 3, pp. 899-918, Apr, 2010.

[2] C. Beels, J.C.C. Henriques, J. De Rouck, M.T. Pontes, G. De Backer, and H. Verhaeghe, "Wave energy resource in the North Sea," $7^{\text {th }}$ European Wave and Tidal Energy Conference, Porto, Portugal, 2007.

[3] J. Weber, F. Mouwen, A. Parrish, and D. Robertson, "Wavebob - Research \& Development Network and Tools in the Context of Systems Engineering," $8^{\text {th }}$ European Wave Tidal Energy Conference, Uppsala, Sweden, 2009.

[4] R. Waters, M. Stalberg, O. Danielsson, O. Svensson, S. Gustafsson, E. Stromstedt, M. Eriksson, J. Sundberg, and M. Leijon, "Experimental results from sea trials of an offshore wave energy system," Applied Physics Letters, vol. 90, no. 3, Jan 15, 2007.

[5] D. Elwood, A. Schacher, K. Rhinefrank, J. Prudell, S. Yim, E. Amon, T. Brekken, and A. von Jouanne, "Numerical Modeling and Ocean Testing of a Direct-Drive Wave Energy Device Utilizing a Permanent Magnet Linear Generator for Power Take-Off," Proceedings of $28^{\text {th }}$ International Conference on Ocean Offshore Arctic Engineering, ASME, Honolulu, Hawaii 2009

[6] E. Tedeschi, and M. Molinas, "Wave-to-Wave Buoys Control for Improved Power Extraction under ElectroMechanical Constraints," IEEE International Conference on Sustainable Energy Technologies (ICSET), Sri Lanka, 2010.

[7] M. Vantorre, R. Banasiak, and R. Verhoeven, "Modelling of hydraulic performance and wave energy extraction by a point absorber in heave," Applied Ocean Research, vol. 26, no. 1-2, pp. 61-72, Feb-Apr, 2004.

[8] G. De Backer, M. Vantorre, R. Banasiak, J. De Rouck, C. Beels, and H. Verhaeghe, "Performance of a point absorber heaving with respect to a floating platform," $7^{\text {th }}$ European Wave and Tidal Energy Conference, Porto, Portugal, 2007. 